\title{
High-frequency viscosity of concentrated porous particles suspensions
}

\author{
Gustavo C. Abade $^{1},{ }^{*}$, Bogdan Cichocki $^{1}$, Maria L. Ekiel-Jeżewska ${ }^{2}{ }^{\dagger}$ \\ Gerhard Nägele ${ }^{3}$, and Eligiusz Wajnryb² \\ ${ }^{1}$ Institute of Theoretical Physics, University of Warsaw, Hoża 69, 00-681 Warsaw, Poland \\ Institute of Fundamental Technological Research, \\ Polish Academy of Sciences, Pawińskiego 5B, 02-106 Warsaw, Poland and \\ ${ }^{3}$ Institut für Festkörperforschung, Forschungszentrum Jülich, D-52425 Jülich, Germany
}

(Dated: June 15, 2010)

\begin{abstract}
We determine the high-frequency limiting shear viscosity, $\eta_{\infty}$, in colloidal suspensions of rigid, uniformly porous spheres of radius $a$ as a function of volume fraction $\phi$ and (inverse) porosity parameter $x$. Our study covers the complete fluid-state regime. The flow inside the spheres is modeled by the Debye-Bueche-Brinkman equation using the boundary condition that fluid velocity and stress change continuously across the sphere surfaces. The many-sphere hydrodynamic interactions in concentrated systems are fully accounted for by a precise hydrodynamic multipole method encoded in our HYDROMULTIPOLE program extended to porous particles. A truncated virial expansion is used to derive an accurate and easy-to-use generalized Saitô formula for $\eta_{\infty}$. The simulation data are used to test the performance of two simplifying effective particle models. The first model describes the effective particle as a non-porous sphere characterized by a single effective radius $a_{\text {eff }}(x)<a$. In the more refined second model, the porous spheres are modeled as spherical annulus particles with an inner hydrodynamic radius $a_{\text {eff }}(x)$ defining the non-porous dry core and characterizing hydrodynamic interactions, and an outer excluded volume radius $a$ characterizing the unchanged direct interactions. Only the second model is in a satisfactory agreement with the simulation data.
\end{abstract}

\footnotetext{
* present address: Departamento de Engenharia Mecânica, Faculdade de Tecnologia, Universidade de Brasília, Campus Universitário Darcy Ribeiro, 70910-900 Brasília-DF, Brazil

${ }^{\dagger}$ Electronic address: mekiel@ippt.gov.pl
} 


\section{INTRODUCTION}

The rheology of suspensions of colloidal particles plays a major role in many technological applications where it is essential to control the flow properties by tuning the shear viscosity. Cost reduction in transportation, e.g., requires the minimization of the viscosity while maintaining the colloid volume fraction as high as possible $[1,2]$.

The suspension rheology is determined by the interplay between the direct particleparticle interactions and the solvent-mediated hydrodynamic interactions (HIs). Calculations of rheological properties such as the low-shear static suspension shear viscosity $\eta$, and the low-shear high-frequency viscosity $\eta_{\infty}$, which take all these interactions into account are additionally complicated by the many-body nature of the HIs in non-dilute systems, and the fact that in many systems the particles are solvent-permeable to some extent. Experimentally studied examples of porous-particle systems are thermosensitive microgel particles [3], soft giant micelles [4] and core-shell particles consisting of a dry solid core, and a solventpermeable thick shell formed by a grafted polymer brush [5]. Solvent-permeability requires to account in addition for the fluid flow inside the particles. This flow changes the strength of the HIs. Since the hydrodynamics of interacting particles with porosity is more complicated, comparatively little theoretical work has been published on the viscosity in dense porous particle systems [6-9]. Most related work has been restricted so far to non-porous particle systems, and foremost here to colloidal hard spheres [10-14]. Simulation results for the high-frequency viscosity of dense suspensions of non-porous charge-stabilized particles with a full account of many-body HIs have been discussed in detail in [15].

To avoid dealing altogether with the complicated HIs, phenomenological approaches such as the differential effective medium theory [16] have been developed. These approaches are based on excluded volume arguments and are often useful from a practical, engineering viewpoint. However, they provide no insight on how the suspension viscosity is precisely controlled by the particle interactions and correlations. For suspensions of non-porous hard spheres, Ref. [17] compares various methods of calculating $\eta_{\infty}$ and $\eta$ to experimental data, and discusses the nature of the divergence of both viscosities at random closed packing.

A common simplifying strategy to include porosity effects is to introduce a porositydependent effective hard-sphere radius, by attempting to map the shear viscosity of porous spheres to that of non-porous ones with a smaller radius and a smaller (effective) volume 
fraction [3]. This crude strategy, which lacks a sound theoretical basis, in general does not lead to satisfactory results, in particular when the effective hard-sphere volume fraction is determined merely by fitting experimental data for the viscosity or diffusion coefficients. Depending on the measured property, different values for the effective hard-sphere radius may be obtained. We point here to a salient difference in the hydrodynamics of porous and non-porous spheres. Different from non-porous spheres with stick boundary conditions, the relative translational mobility of two porous spheres in a squeezing motion along their line of centers is non-zero at contact [18], since the fluid can penetrate the particles. Two important consequences of non-zero porosity are an enhanced coagulation kinetics of attractive porous particles in unstable suspensions [19], and the suppression of lubrication-based hydro-clusters in concentrated colloidal suspensions subject to strong shear flow. By the term hydrocluster one refers to jamming clusters of colloidal particles bound together by hydrodynamic lubrication forces, which can form in dense suspensions under increasing shear rates. These clusters cause strong, reversible shear thickening, taken advantage of recently in the design of soft body armor composites [20].

An alternative simplifying route to compute the shear viscosity of dense porous particle systems, followed in particular by Ohshima and collaborators [21, 22], are spherical cell model calculations. The cell model is frequently used to study the primary electroviscous effect on the high-frequency viscosity in non-dilute suspensions of porous [23] and non-porous [24] charged spheres with weakly overlapping double layers. While the single-sphere porosity is correctly accounted for in this model, inter-particle correlations arising from hydrodynamic and electro-steric interactions are considered very crudely only through the concentration dependence of the outer spherical radius and the specified boundary conditions. At larger concentrations where fluid-like particle correlations are important, the cell model makes poor predictions for the high-frequency viscosity [25].

In the present article, we are concerned with the low-shear high-frequency viscosity, $\eta_{\infty}$, of a monodisperse suspension of non-overlapping porous spherical particles as a function of volume fraction and porosity. High-frequency means here that a low-amplitude oscillating shear stress of such a high frequency is applied to the system that the microstructure of the suspension has no time to relax by diffusion during the cycle. The system remains locally isotropic in its unperturbed equilibrium state in spite of the applied stress. Thus, $\eta_{\infty}$ is directly influenced by the instantaneous HIs only. The non-hydrodynamic (excluded vol- 
ume) forces contribute only indirectly by determining the equilibrium particle distribution. Consequently, in contrast to the static (zero-frequency) viscosity $\eta$ which is also directly influenced by the non-hydrodynamic forces so that additional viscous dissipation occurs, leading to $\eta>\eta_{\infty}, \eta_{\infty}$ should be only mildly dependent on the nature and strength of these forces. In fact, the high-frequency viscosity of suspensions of (non-porous) charged and neutral particles have been shown to behave quite similarly [15, 26, 27].

High-frequency viscoelastic measurements using, e.g., a torsional resonator have become standard, and have been made for a variety of colloidal systems [26-29]. To measure the zero-shear-rate limiting high-frequency viscosity considered in this work, the experimentally applied stress amplitude should be small enough to avoid significant shear-thinning. For particulate systems where the amount of available colloids is too low to perform a mechanical rheological experiment, one can try to infer $\eta_{\infty}$ from a microrheological scattering experiment which relies on the approximate validity of a generalized Stokes-Einstein (GSE) relation. Simulation $[15,25]$ and experimental $[30,31]$ tests of GSE relations between $\eta_{\infty}$ and various short-time diffusion coefficients have demonstrated that the accuracy of a specific GSE relation depends strongly on the form, and in particular on the range of the pair potential. For neutral porous spheres, we have recently shown by simulations that the GSE relation relating $\eta_{\infty}$ to the short-time cage diffusion coefficient, a quantity which can be easily measured in a dynamic scattering experiment by a first cumulant analysis, provides an estimate for $\eta_{\infty}$ of accuracy better than $20 \%$ [25].

An individual particle is modeled in our study as a rigid sphere of (excluded volume) radius $a$ and constant Darcy porosity $k$, with the fluid flow inside the particle described by the Debye-Bueche-Brinkman equation [32-38]. This basic model allows to study generic porosity effects using a minimal number of parameters. It ignores more specific features such as a non-uniform intra-particle porosity distribution and a flexible particle shape playing a role, e.g., in soft giant micellar systems. The particle porosity in typical colloidal systems is not large. Thus, a non-uniform porosity distribution such as in core-shell particles, can be accounted for to a decent approximation using an average porosity value. Also, in addition to the excluded volume interaction, we do not consider here (soft) pair interaction contributions such as electric double layer repulsion. Actually, the influence of porosity is smaller for charge-stabilized particles, since near-contact configurations, where intra-particle flow is most influential, are very unlikely. 
The many-sphere hydrodynamic interactions in a concentrated system of porous spheres are fully accounted for by our precise hydrodynamic multipole method (see [39-42] for details) encoded in the HYDROMULTIPOLE program which we have extended by the inclusion of particle porosity $[43,44]$. Using this method, we have obtained data for $\eta_{\infty}$ for volume fractions $\phi$ covering the equilibrium fluid phase regime. The considered porosity values span the range from non-porous to highly porous particles. We perform a virial expansion of $\eta_{\infty}$ up to third order in the volume fraction. This expansion is used to derive a convenient extended Saitô formula for $\eta_{\infty}$, which accurately applies to fluid-ordered systems.

In addition, our simulation data for $\eta_{\infty}$ are used to probe the accuracy of two simplifying effective particle models. In the first model, referred to as the effective radius model (ERM), the porous particles of radius $a$ are approximately described as non-porous spheres with a porosity-dependent effective radius $a_{\text {eff }}<a$, and a corresponding effective volume fraction $\phi_{\text {eff }}=\phi\left(a_{\mathrm{eff}} / a\right)^{3}$. In the more refined second model, referred to as the hydrodynamic radius model (HRM), a porous sphere is described as a spherical annulus particle with inner hydrodynamic radius $a_{\text {eff }}<a$ of a non-porous core, and the unchanged direct interaction radius $a$. The hydrodynamic core radius in the HRM is equal to the effective radius used in the ERM. As we will show, only the second model is in satisfactory agreement with the simulation data.

The paper is organized as follows: Section II describes the model of porous spheres and provides the theoretical background for the numerical calculation of $\eta_{\infty}$ by our hydrodynamic multipole method. Simulation results for $\eta_{\infty}$ are discussed in Sec. III. This section additionally includes viscosity results obtained from the virial expansion and the generalized Saitô formula. The effective particle models are explained and tested against simulations in Sec. IV. Our conclusions are contained in Sec. V.

\section{SYSTEM AND THEORETICAL BACKGROUND}

\section{A. System}

We consider a suspension of freely moving, identical spherical particles with radius $a$ and Darcy porosity $k$, immersed in an unbound Newtonian fluid of shear viscosity $\eta_{0}$. Moreover we assume a hard-sphere direct interaction corresponding to the same radius $a$. The creeping 
solvent flow outside the homogeneously porous and non-deformable spheres is described by the Stokes equation [45]

$$
\boldsymbol{\nabla} \cdot \boldsymbol{\sigma}(\mathbf{r})=0
$$

and inside by the Debye-Bueche-Brinkman (DBB) equation [32-37]

$$
\boldsymbol{\nabla} \cdot \boldsymbol{\sigma}(\mathbf{r})=\eta_{0} \kappa^{2}\left(\boldsymbol{v}(\boldsymbol{r})-\boldsymbol{u}_{i}(\boldsymbol{r})\right)
$$

respectively, where $i \in\{1, \ldots, N\}$ is the particle index and $N$ denotes the number of particles in a volume $V$. Here,

$$
\boldsymbol{\sigma}(\mathbf{r})=-p(\boldsymbol{r}) \mathbf{1}+\eta_{0}\left(\boldsymbol{\nabla} \boldsymbol{v}(\boldsymbol{r})+(\boldsymbol{\nabla} \boldsymbol{v}(\boldsymbol{r}))^{T}\right)
$$

is the Newtonian stress tensor of an incompressible fluid characterized by $\boldsymbol{\nabla} \cdot \boldsymbol{v}(\boldsymbol{r})=0$, and the index $T$ stands for the matrix transposition.

Furthermore, $\boldsymbol{v}$ and $p$ are the fluid velocity and pressure fields, and $\kappa^{-1}=\sqrt{k}$ is the hydrodynamic penetration depth. Inside the spheres, $\boldsymbol{v}$ and $p$ should be interpreted as pore-size distribution averaged quantities. The porous skeleton of a sphere $i$ moves rigidly according to $\mathbf{u}_{i}(\mathbf{r})=\mathbf{U}_{i}+\boldsymbol{\omega}_{i} \times\left(\mathbf{r}-\mathbf{R}_{i}\right)$, with the translational and rotational velocities $\mathbf{U}_{i}$ and $\boldsymbol{\omega}_{i}$, respectively. The relative motion of the skeleton and the intra-particle flow creates a friction force density proportional to $\boldsymbol{v}-\boldsymbol{u}_{i}$. In the zero-porosity limit $\kappa \rightarrow \infty, \boldsymbol{v}(\mathbf{r})=\mathbf{u}_{i}(\mathbf{r})$ inside a sphere $i$, so that spheres with stick surface boundary conditions are described.

To determine $\eta_{\infty}$ and other transport properties, requires to solve the flow equations inside and outside the spheres, with the boundary conditions that $\boldsymbol{v}$ and the solvent stress tensor components (and thus the fluid pressure) change continuously across the sphere surfaces [38]. The DBB equation for the intra-particle flow applies when the mean pore size $\delta$ of the skeleton, with $\delta \sim \kappa^{-1}$, is sufficiently smaller than the particle radius $a$, i.e., when the dimensionless inverse penetration depth, $x=\kappa a$, is sufficiently large (i.e., $x \gtrsim 5$ ). In the zero-penetration limit $x \rightarrow \infty$, hard spheres with stick boundary conditions at the surface are recovered. The model of homogeneously porous spheres is completely characterized by $x$ and the particle volume fraction $\phi=4 \pi a^{3} n / 3$, the latter determined by the radius $a$ and the particle number concentration $n=N / V$. In contrast to non-porous rigid spheres with stick surface boundary conditions at the surface, the relative mobility for the squeezing (shearing) motion of two porous spheres along (perpendicular) their line of centers is nonzero at contact. 


\section{B. Theoretical background}

In this subsection we describe how the high-frequency shear mobility of an isotropic suspension of freely mobile particles is calculated. The transport coefficient $\eta_{\infty}$ linearly relates the average deviatoric suspension stress to the average high-frequency oscillatory rate of strain. To obtain $\eta_{\infty}$ in our simulations, we consider $N$ force and torque free spheres in a periodically replicated simulation box of volume $V$. The statistical mechanical definition reads then [46],

$$
\eta_{\infty}=\eta_{0}+\frac{1}{10 V}\left\langle\sum_{i, j=1}^{N} \mu_{i j, \alpha \beta \beta \alpha}\right\rangle^{p b c}
$$

where $\mu_{i j, \alpha \beta \gamma \delta}$ are the Cartesian components of three-dimensional, fourth-rank dipole-dipole mobility tensors, $\boldsymbol{\mu}_{i j}$, which depend on the instantaneous configuration of all particles. The dipole-dipole tensors linearly relate the stresslets, i.e.,the symmetric hydrodynamic force dipole moments, $\mathbf{S}_{i}$, acting on the surfaces of spheres $i=1, \cdots, N$, to the 2 nd rank rate of strain tensors $\mathbf{g}_{j}$,

$$
\mathbf{S}_{i}=\sum_{j=1}^{N} \boldsymbol{\mu}_{i j} \cdot \mathbf{g}_{j}
$$

The strain tensors describe the linear shear flow incident on spheres $j=1, \cdots, N$. For notational simplicity, we have omitted the "dd" superscript commonly assigned to the dipoledipole mobility tensor $\boldsymbol{\mu}_{i j}[39,47]$. In Eq. (4), the Einstein summation convention is implied for the Cartesian components $\alpha$ and $\beta$.

The fourth-rank tensors $\boldsymbol{\mu}_{i j}$, are evaluated from the multipole expansion method (see, e.g., [46-48]). In general, the average $\langle\ldots\rangle^{p b c}$ can be taken over an arbitrary ensemble of non-overlapping random configurations, consistent with periodic boundary conditions. In the linear response regime considered in this paper, we use the isotropic equilibrium particle distribution characterizing a fluid system.

At small volume fractions $\phi, \eta_{\infty}$ can be expanded in a virial series as [48]

$$
\eta_{\infty}(x, \phi)=\eta_{0}\left(1+[\eta] \phi+k_{H}[\eta]^{2} \phi^{2}+\mathcal{O}\left(\phi^{3}\right)\right) .
$$

Here, $[\eta]$ is the intrinsic viscosity, a single-particle property depending on the reduced penetration depth $x$. The intrinsic viscosity of homogeneously porous, rigid spheres is given by $[35,37]$

$$
[\eta]=\frac{5}{2} \Omega_{v}(x)=\frac{5}{2}\left(\frac{G(x)}{1+10 G(x) / x^{2}}\right),
$$


with

$$
G(x)=1+\frac{3}{x^{2}}-\frac{3 \operatorname{coth}(x)}{x} .
$$

In the limit $x \rightarrow \infty,[\eta]$ attains its maximal value $5 / 2$, which is Einstein's famous result for the intrinsic viscosity of non-porous rigid spheres with stick surface boundary conditions. The porosity dependence of $[\eta]$ reflects the lower viscous dissipation caused by porous particles, as compared to a non-porous ones, since the solvent can flow through them. This is also valid for higher volume fractions where inter-particle HIs have to be considered which are weakened in the presence of porosity. Consequently, the high-frequency viscosity becomes smaller with increasing porosity (decreasing $x$ ). Note that in Eq. (6), we have followed the standard convention by formulating the virial series as an expansion in powers of $[\eta] \phi$.

The Huggins coefficient, $k_{H}(x)$, introduced in Eq. (6), accounts for two-body hydrodynamic interactions. For spheres of radius $a$, this coefficient can be calculated from [49-51],

$$
k_{H}=\frac{2}{5}+\frac{3}{[\eta] a^{3}} \int_{2 a}^{\infty} g_{0}(r) J(r) r^{2} d r
$$

with

$$
J(r)=\frac{3}{40 \pi \eta_{0}[\eta] a^{3}}\left[\mu_{11, \alpha \beta \beta \alpha}^{(2)}(\mathbf{r})+\mu_{12, \alpha \beta \beta \alpha}^{(2)}(\mathbf{r})\right],
$$

where $\mathbf{r}$ is the relative position vector of magnitude $r$ joining the centers of two particles, and $g_{0}(r)=\exp \{-\beta u(r)\}$ is the pair correlation function at infinite dilution expressed in terms of the pair potential $u(r)$. For the equilibrium distribution of hard spheres with excluded volume of radius $a, g_{0}(r)=\theta(r-2 a)$ where $\theta(r)$ is the unit step function.

According to Eq. (9), the Huggins coefficient consists of a microstructure-independent universal part equal to $2 / 5$, and a contribution depending on the pair structure. The dipoledipole tensor elements $\boldsymbol{\mu}_{11}^{(2)}(\mathbf{r})$ and $\boldsymbol{\mu}_{12}^{(2)}(\mathbf{r})$ in Eq. (10) are the two-body cluster parts of the full many-body dipole-dipole tensor in Eq. (4) (see [47]). The isotropic function $J(r)=$ $J(r / a, x)$, first introduced by Batchelor and Green for the special case of non-porous hard spheres [49], is rather short-ranged, decaying asymptotically like $r^{-6}$.

\section{RESULTS}

We have calculated to high precision both $k_{H}$ (from the series expansion of $J(r)$ in inverse powers of $r$ ) and $\eta_{\infty}$ (using a hydrodynamic multipole method corrected for 
lubrication [39-42], encoded in our HYDROMULTIPOLE program package extended to porous spheres [43, 44]). The hydrodynamic structure of the particles enters into the HYDROMULTIPOLE code through a single-particle friction operator only, whose form is known for a variety of particle models with different internal hydrodynamic structures $[39,52]$. The values of $\eta_{\infty}$ presented in this paper have been obtained from equilibrium configuration averages over typically $N=256$ particles in a periodically replicated cubic simulation box, with the hydrodynamic multipole order $L$ truncated usually at $L=3$. To gain high-precision data, an extrapolation procedure to $L \rightarrow \infty$ has been applied. It was pointed out already by Ladd [10], and explained by Mo and Sangani [6], that the simulated values for $\eta_{\infty}$ are not critically dependent on finite size scaling, and extrapolation to the thermodynamic limit $N \rightarrow \infty$ is not needed. In our simulations, the remaining error in $\eta_{\infty}$ is less than $1 \%$.

\section{A. Simulation data}

In table I, our simulation results for the concentration dependence of $\eta_{\infty} / \eta_{0}$, are listed for a wide range of $x$ values.

For non-zero porosity $(x<\infty)$, the penetrating fluid can flow through the particles. This partially relieves the local stress in the fluid performing a straining motion. The stress and viscous dissipation in the fluid caused by the particles is thus smaller at larger porosity so that $\eta_{\infty}$ becomes smaller with increasing porosity (decreasing $x$ ). The decrease in the high-frequency viscosity with increasing porosity is substantial at larger $\phi$ and should be easily detectable experimentally. For instance, in a dispersion of particles at $\phi=0.35$ with a typical mean porosity of $x \approx 30$ [5], the viscosity is about $30 \%$ lower than at zero porosity.

Our simulations results for $\eta_{\infty} / \eta_{0}$ are in agreement with those of Mo and Sangani [6] in the more restricted range of $x \leq 20$ values considered in their study, which is based on a multipole expansion method different from ours. For porous particles with $x \leq 20$, the largest differences between their values and our findings, detected at higher volume fractions, are of the order of $1 \%$. Slightly larger differences are expected for larger values of $x$. For non-porous hard spheres where $x=\infty$, and $\phi=0.35$ and 0.45 , the results in Ref. [6] underestimate $\eta_{\infty} / \eta_{0}$ by $6 \%$ and $4 \%$, respectively. This inaccuracy can be attributed to 
TABLE I: Reduced high-frequency viscosity $\eta_{\infty} / \eta_{0}$, as a function of $\phi$ and reduced inverse penetration depth $x$.

\begin{tabular}{|c|c|c|c|c|c|c|c|}
\hline$\phi \backslash x$ & 5 & 10 & 20 & 30 & 50 & 100 & $\infty$ \\
\hline 0.05 & 1.055 & 1.090 & 1.113 & 1.122 & 1.129 & 1.134 & 1.139 \\
\hline 0.15 & 1.176 & 1.301 & 1.399 & 1.438 & 1.472 & 1.499 & 1.527 \\
\hline 0.25 & 1.311 & 1.565 & 1.794 & 1.897 & 1.994 & 2.08 & 2.17 \\
\hline 0.35 & 1.462 & 1.893 & 2.35 & 2.58 & 2.82 & 3.04 & 3.33 \\
\hline 0.45 & 1.629 & 2.30 & 3.13 & 3.61 & 4.17 & 4.76 & 5.65 \\
\hline
\end{tabular}

the very small number, $N=16$, of particles per basic simulation cell used in their study. This number is too low to obtain equilibrium correlations with a high precision. On the other hand, our viscosity data for the limiting case of non-porous spheres agree well with earlier force multipole simulation data by Ladd [10], who used a larger numbers, $N \leq 108$, of particles, and with the accelerated Stokesian dynamics simulation data by Sierou and Brady [13] and Banchio and Nägele [15].

\section{B. Virial expansion and generalized Saitô approximation}

The Huggins coefficient, $k_{H}(x)$, is determined by two-body hydrodynamic contributions as described by Eq. (9). For non-porous hard spheres where $[\eta]=5 / 2$, Batchelor and Green [49] obtained $k_{H}(\infty)[\eta]^{2}=5.2$, an approximate value that was improved in later work by Cichocki and Felderhof [51] to the high-precision result of 5.00. Data for the Huggins coefficient of porous spheres have not been presented so far. Table II fills this gap by displaying our calculated values for this coefficient as a function of $x$ [25]. The corresponding values of the intrinsic viscosity, $[\eta]$, and total second-order virial coefficient $k_{H}[\eta]^{2}$ (see Eq. (6)) are also included for comparison. 
TABLE II: Huggins coefficient, $k_{H}$, and the second virial coefficient, $k_{H}[\eta]^{2}$, as functions of $x$. The intrinsic viscosity $[\eta]$ is shown for a comparison.

\begin{tabular}{cccccccc}
\hline \hline $\mathrm{x}$ & 5 & 10 & 20 & 30 & 50 & 100 & $\infty$ \\
\hline$[\eta]$ & 1.076 & 1.701 & 2.099 & 2.236 & 2.344 & 2.423 & 2.500 \\
$k_{H}$ & 0.5321 & 0.6270 & 0.7019 & 0.7323 & 0.7587 & 0.7796 & 0.8004 \\
$k_{H}[\eta]^{2}$ & 0.6161 & 1.814 & 3.092 & 3.661 & 4.169 & 4.578 & 5.002 \\
\hline \hline
\end{tabular}

By taking advantage of our results for $k_{H}(x)$, we can obtain an approximate analytic expression for $\eta_{\infty}$, valid to larger $\phi$ than the 2nd-order (in concentration) virial expansion result, from the generalized Saitô formula [48]

$$
\frac{\eta_{\infty}}{\eta_{0}}=1+[\eta] \phi \frac{1+S}{1-\frac{2}{5}[\eta] \phi(1+S)},
$$

which expresses $\eta_{\infty}$ in terms of the intrinsic viscosity $[\eta]$ and a function $S(x, \phi)$, referred to in the following as the Saitô function. An exact but formal microscopic expression for the Saitô function of spherical particles and droplets has been derived in [48]. Since $S$ is more amenable to a low-concentration approximation than $\eta_{\infty}$ itself, in a first step we determine it approximately by equating the first-order in concentration expansions of Eqs. (11) and (6). This leads to a useful first-order in concentration approximation for $S$,

$$
S_{1}(x, \phi)=b_{1}(x) \phi
$$

with

$$
b_{1}(x)=\left(k_{H}-\frac{2}{5}\right)[\eta]
$$

in terms of the one-body and two-body properties $[\eta]$ and $k_{H}$, which both depend on $x$ only.

In Fig. 1, the high-frequency viscosity calculated by the HYDROMULTIPOLE numerical code (symbols) is compared with theoretical approximations: the Saitô and virial expressions (the top and bottom panels, respectively). The top panel (dashed lines) shows the viscosity prediction by the generalized Saitô formula in Eq. (11) in combination with the first-order Saitô function $S_{1}(x, \phi)$ in Eq. (12) with $[\eta]$ according to Eq. (7), and values for $k_{H}(x)$ taken from table I. For the whole range $\phi \leq 0.45$, there is good overall agreement with the simulation data for $\eta_{\infty}$, in particular for very small and large values of $x$. However, at intermediate $x$ and larger $\phi$, the approximate viscosity is somewhat overestimated. 

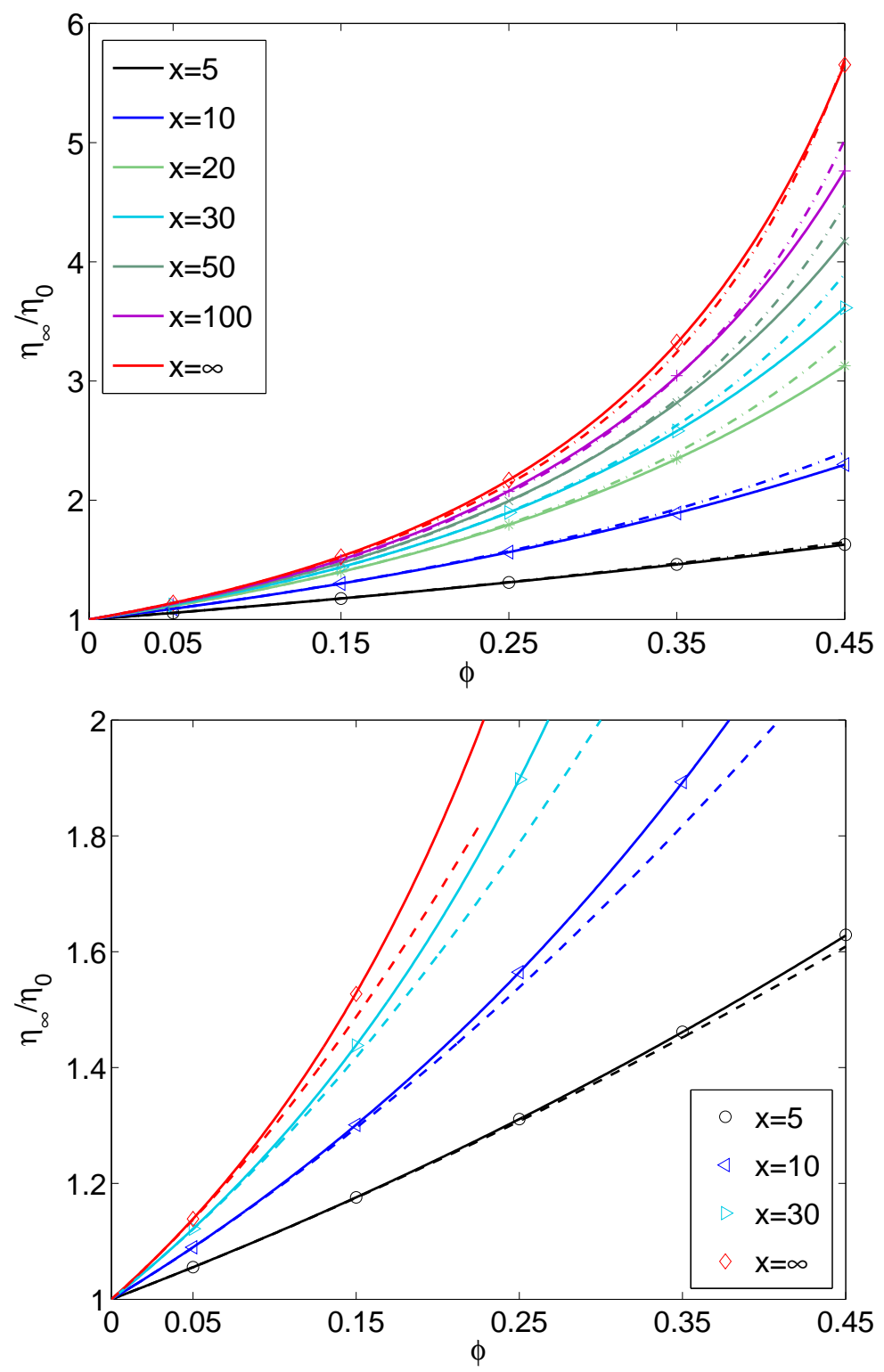

FIG. 1: Low-shear high-frequency viscosity, $\eta_{\infty} / \eta_{0}$, of uniformly porous spheres for porosities as indicated ( $x=\infty$ corresponds to non-porous hard spheres). Simulation results (symbols), fitted by solid lines, versus theoretical approximations. The fitting is performed using Eqs. (11) and (14). Top: Comparison with the generalized Saitô formula in Eqs. (11)-(12) (dashed-dotted lines). Bottom: Comparison with the second-order in $\phi$ viral expansion result for $\eta_{\infty}$ in Eq. (6) (dashed lines).

That the generalized Saitô formula is more useful than the 2nd-order virial expansion result for $\eta_{\infty}$, even though the same input $[\eta]$ and $k_{H}$ is used, can be clearly noticed from Fig. 1 (bottom panel). The simulation results for $\phi>0.2$ are only well reproduced by the 
TABLE III: Coefficients of the third-order in concentration Saitô function, $S_{3}(x, \phi)$, defined by Eq. (14), with $b_{1}$ given by (13) and $b_{2}, b_{3}$ fitted to the numerical results.

\begin{tabular}{clcc}
\hline \hline $\mathrm{x}$ & $b_{1}$ & \multicolumn{1}{c}{$b_{2}$} & $b_{3}$ \\
\hline 5 & 0.142 & -0.10 & -0.08 \\
10 & 0.386 & -0.13 & -0.34 \\
20 & 0.634 & 0.07 & -0.90 \\
30 & 0.743 & 0.25 & -1.25 \\
50 & 0.841 & 0.46 & -1.59 \\
100 & 0.920 & 0.69 & -1.93 \\
$\infty$ & 1.001 & 0.95 & -2.15 \\
\hline \hline
\end{tabular}

2nd-order virial form for large porosities, i.e., small values of $x$. This is a consequence of the decreasing strength of the three-body and higher-order hydrodynamic contributions to $\eta_{\infty}$ with increasing porosity.

The remaining differences between the simulation data and the generalized Saitô formula result for $\eta_{\infty}$ with $S_{1}(x, \phi)$ as input, can be remedied when, in place of $S_{1}(x, \phi)$, a third-order in $\phi$ polynomial fit of the Saiô function is used,

$$
S_{3}(x, \phi)=b_{1}(x) \phi+b_{2}(x) \phi^{2}+b_{3}(x) \phi^{3}
$$

with $b_{1}(x)$ given by Eq. (13). The coefficients $b_{2}(x)$ and $b_{3}(x)$, obtained from a fit of the simulation data for $\eta_{\infty}$ in the range $\phi \leq 0.45$, are given in table III for a representative set of $x$ values. Also, $b_{1}(x)$ is listed for a comparison. The high-frequency viscosity obtained from the simple expressions, Eq. (11) in conjunction with Eq. (14), reproduces very accurately the fluid-state simulation data for all considered $x$ values, as shown in Fig. 1 (solid lines).

In the zero-porosity limit, table III gives

$$
S_{3}(\infty, \phi)=1.001 \phi+0.95 \phi^{2}-2.15 \phi^{3}
$$

This expression for the Saitô function of non-porous spheres is very similar, albeit not identical, to the expression

$$
S_{\text {Ladd }}(\infty, \phi)=\phi+\phi^{2}-2.3 \phi^{3}
$$


which was used by Ladd [10] in conjunction with the generalized Saitô formula to fit his simulation data for viscosity $\eta_{\infty}(\infty, \phi)$ of hard non-porous spheres.

While the present work was focused on the high-frequency viscosity of porous spheres in the equilibrium fluid regime $(\phi<0.494)$, a comment is in order here on the expected behavior of $\eta_{\infty}$ and $\eta$ in the more concentrated metastable fluid regime limited by the random closed packing volume fraction value $\phi_{\mathrm{rcp}} \approx 0.64$. Like for non-porous hard spheres $[13,17,53]$, both $\eta_{\infty}(x, \phi)$ and the static viscosity $\eta(x, \phi)$ are expected to cross over smoothly, with increasing volume fraction, from the stable to the metastable fluid regime. For non-porous spheres with stick surface boundary conditions, both low-shear viscosities, $\eta$ and $\eta_{\infty}$, diverge at random closed packing, since the rigid spheres are jammed in their relative positions, unable to move translationally and rotationally. For non-zero porosity, however, $\eta_{\infty}$ is not divergent any more. The spheres can still rotate even though translationally immobilized, and the fluid can still flow through the particles (see also [20]). For porous and non-porous spheres alike, the divergence of $\eta$ at random closed packing may be preempted by a possible divergence at the glass transition volume fraction $\phi_{\mathrm{g}} \approx 0.58[17,53]$. Although the presented generalized Saitô formulas for $\eta_{\infty}$ are based on simulation data for the stable fluid phase, it is worth while to note their respective predictions on the divergence of $\eta_{\infty}(\infty, \phi)$ in the metastable regime. The generalized Saitô formula combined with $S_{3}(\infty, \phi)$, which accurately describes $\eta_{\infty}$ in the equilibrium fluid regime, predicts its divergence at $\phi \approx 0.70$, a volume fraction somewhat larger than $\phi_{\text {rcp }}$. When Ladd's third-order polynomial $S_{3}(\infty, \phi)$ is used instead, the divergence of $\eta_{\infty}$ is predicted at $\phi \approx 0.73$. The less accurate first-order Saitô function $S_{1}(\infty, \phi)$ predicts divergence at $\phi \approx 0.62$, close to $\phi_{\text {rcp }}$. However, this result is fortuitous since, as noted earlier, $S_{1}(x, \phi)$ and $S_{3}(x, \phi)$ have been obtained from simulation data in the equilibrium fluid phase alone.

\section{EFFECTIVE MODELS}

In this section, we discuss simple routes to calculate approximately the high-frequency viscosity of concentrated suspensions of porous spheres, using known analytic results for the hydrodynamics of an isolated porous sphere, together with known results and standard methods for the high-frequency viscosity of non-porous, interacting spheres.

We start by noting that $\Omega_{v}(x)$ in Eq. (7) can be used $[54,55]$ to define a porosity- 
dependent, effective radius $a_{\text {eff }}<a$, with

$$
\frac{a_{\mathrm{eff}}(x)}{a}=\left[\Omega_{v}(x)\right]^{1 / 3},
$$

describing non-porous hard spheres with stick hydrodynamic boundary conditions at the smaller effective radius $a_{\text {eff. }}$ Since

$$
\frac{5}{2} \phi_{\mathrm{eff}}=[\eta] \phi
$$

where $\phi_{\text {eff }}(x)=\phi \Omega_{v}(x)<\phi$ is the volume fraction of the non-porous hard spheres, one notices from Eq. (6) that, to first order in concentration, the suspension of non-porous smaller spheres has the same viscosity as the actual suspension of porous-spheres.

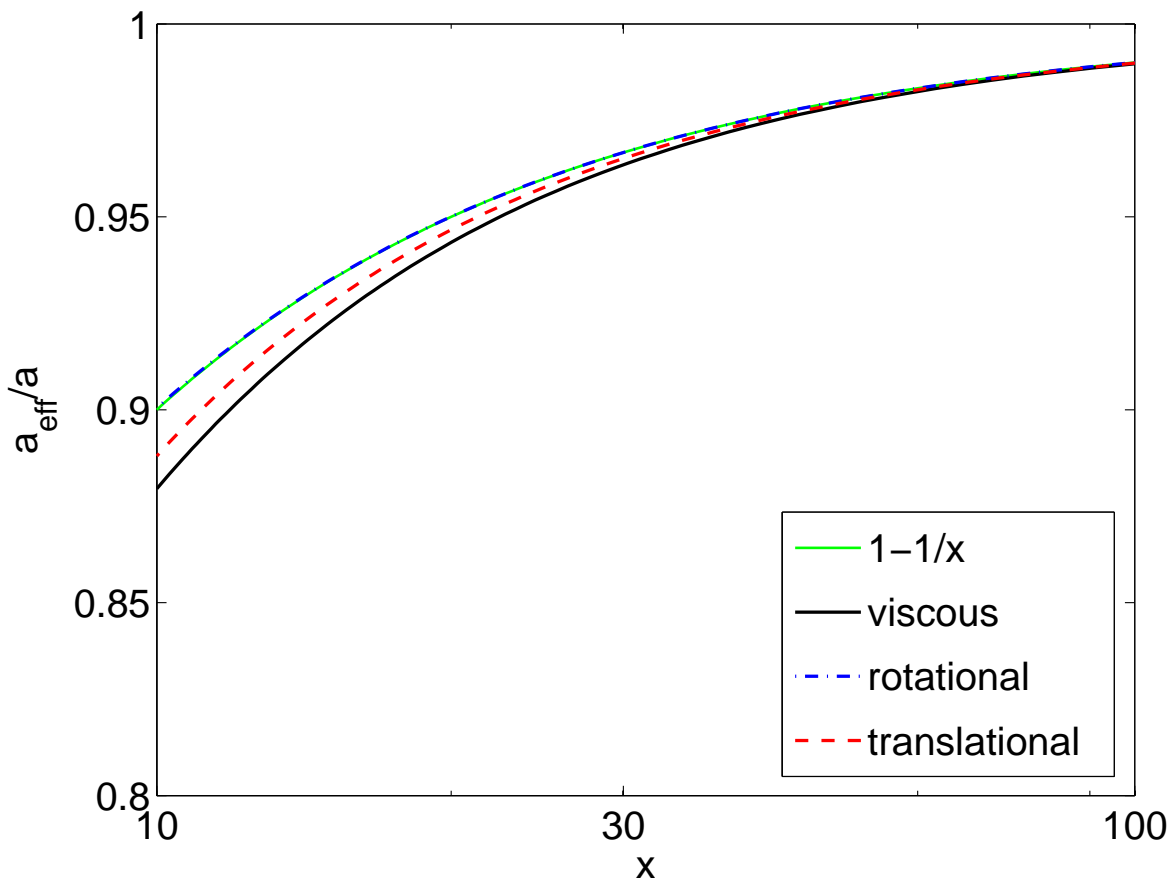

FIG. 2: Semilogarithmic plot of three different single-sphere hydrodynamic radii. For $x>20$, the common asymptotic form $1-1 / x$ is practically reached.

One can easily show that

$$
\frac{a_{\mathrm{eff}}(x)}{a}=1-\frac{1}{x}+\mathcal{O}\left(\frac{1}{x^{2}}\right)
$$

for lower porosity where $x \gg 1$. Quite interestingly, the very same leading-order expansion in $1 / x$ is obtained for two alternative definitions of effective radii, $a_{\mathrm{eff}}^{t}(x) / a$ and $a_{\mathrm{eff}}^{r}(x) / a$, 
given by the single-sphere Stokes-Einstein relations

$$
\begin{aligned}
& D_{0}^{t}(x)=\frac{k_{B} T}{6 \pi \eta_{0} a_{\mathrm{eff}}^{t}(x)} \\
& D_{0}^{r}(x)=\frac{k_{B} T}{8 \pi \eta_{0}\left(a_{\mathrm{eff}}^{r}(x)\right)^{3}},
\end{aligned}
$$

where $D_{0}^{t}(x)$ and $D_{0}^{r}(x)$ are, respectively, the translational and rotational diffusion coefficients of an isolated, uniformly porous sphere. The expression for $D_{0}^{t}(x)$ was first derived by Brinkman [35] and Debye and Bueche [37]. Substitution of this expression into Eq. (20), solved for $a_{\text {eff }}^{t}$ gives

$$
\frac{a_{\mathrm{eff}}^{t}(x)}{a}=\frac{2 x^{2}(x-\tanh (x))}{2 x^{3}+3(x-\tanh (x))} .
$$

The corresponding expression for $D_{t}^{r}(x)$ was derived by Felderhof et al. [56, 57], leading in combination with Eq. (21) to

$$
\frac{a_{\mathrm{eff}}^{r}(x)}{a}=[G(x)]^{1 / 3}
$$

with $G(x)$ defined in Eq. (8). The three hydrodynamic radii $a_{\mathrm{eff}}, a_{\mathrm{eff}}^{t}$ and $a_{\mathrm{eff}}^{r}$ characterizing a porous sphere of radius $a$, are defined as the radii of a non-porous effective sphere sharing the same hydrodynamic property which is, respectively, the first-order in density viscosity, the single-particle translational friction coefficient and the single-particle rotational friction coefficient. Fig. 2 displays the three hydrodynamic radii as functions of $x$. Clearly, the radii are not identical, and their differences increase with increasing porosity. However, they share the same large- $x$ asymptotic form given in Eq. (19), with differences due to the small $\mathcal{O}\left(x^{-2}\right)$ corrections only. For the majority of experimentally studied porous particles, $x$ is greater than 20, so that the effective radius defined by Eq. (17) is unique for all practical purposes.

The fact that the considered effective radii are identical to first order in $1 / x$ is not accidental. The reason for this is that the Stokes flow at a long distance $d$ from a weakly porous wall is the same as when the wall is replaced by a stick-boundary wall at a slightly larger distance $d+l[58-60]$. The displacement, or effective slip distance, $l$ is independent of the form of the Stokes flow. In the present problem, $l \sim a-a_{\mathrm{eff}}$, given that $l / a$ is small, i.e., given that $x$ is sufficiently large. 


\section{A. Effective radius model}

In the most simple approach using the concept of an effective hydrodynamic radius, the porous particles of radius $a$ are described approximately as non-porous hard spheres of a smaller effective radius $a_{\text {eff }}(x)$ given by Eq. (17). In this simple effective radius model (ERM) (see Fig. 3), the high-frequency viscosity is given by

$$
\eta_{\infty}^{\mathrm{ERM}}(x, \phi)=\eta_{\infty}\left(\infty, \phi_{\mathrm{eff}}\right)
$$

with $\phi_{\text {eff }}$ expressed in terms of $\phi$ by Eq. (18). The right-hand-side of Eq. (24) can be readily evaluated from Eqs. (11) and (16), with $\phi_{\text {eff }}$ substituted for $\phi$.

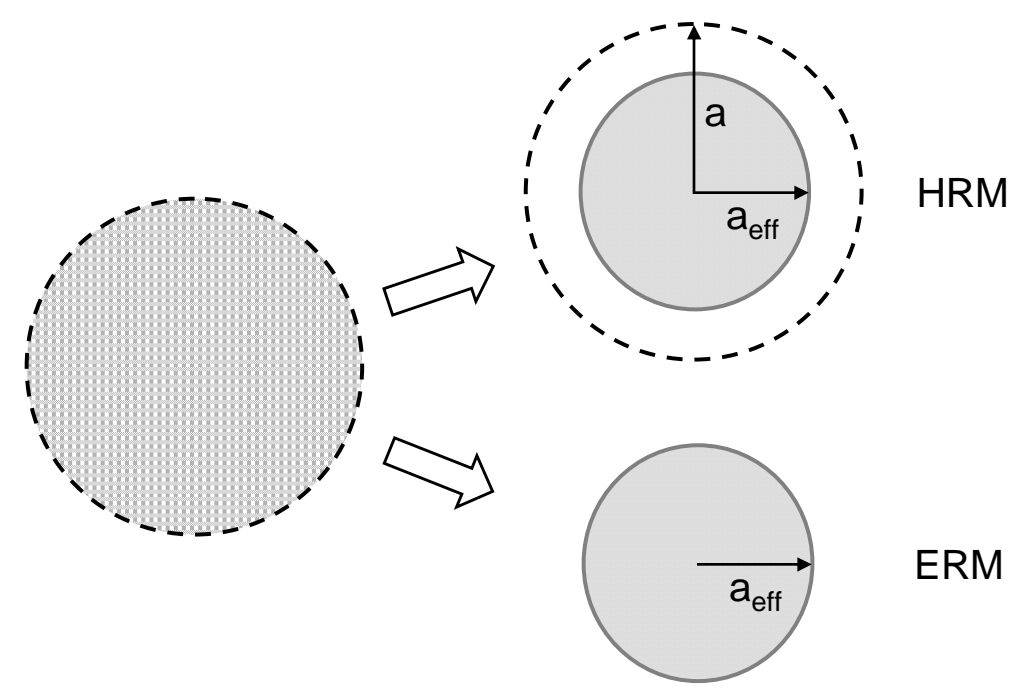

FIG. 3: In the effective radius model (ERM), a uniformly porous sphere of radius $a$ is approximated by a smaller, non-porous hard sphere of radius $a_{\text {eff }}(x)$ defined in Eq. (17). In the more refined hydrodynamic radius model (HRM), the porous sphere is approximated by a spherical annulus particle consisting of a non-porous core of hydrodynamic radius $a_{\mathrm{eff}}(x)$, and an unchanged outer excluded volume radius $a$.

By its definition, this simplifying model works well for dilute systems where the interparticle correlations are weak. Its accuracy is expected to deteriorate at higher $\phi$. To understand the reason, note that in this model, the statistical distribution of the nonporous effective particles is obtained from rescaling all distances by $a_{\mathrm{eff}}(x) / a$. It follows, in particular, that in the ERM model $g_{0}(r)$ is given as $\theta\left(r-2 a_{\mathrm{eff}}(x)\right)$. From the substitution of $\phi_{\text {eff }}$ into the virial expansion of non-porous spheres, and from recalling Eq. (18), it follows 
that already the second virial coefficient is in general not accurately reproduced in this model, since $k_{H}(x)$ is approximated for all $x$ by the zero-porosity value $k_{H}(\infty)=0.8004$. For larger porosity, however, $k_{H}(\infty)$ differs significantly from the actual Huggins coefficient $k_{H}(x)$ of porous spheres listed in table I, with a relative difference of $15-10 \%$ for typical values $x=20-30$. In this range of intermediate and large porosity values, the second virial coefficient differs from its ERM approximation.

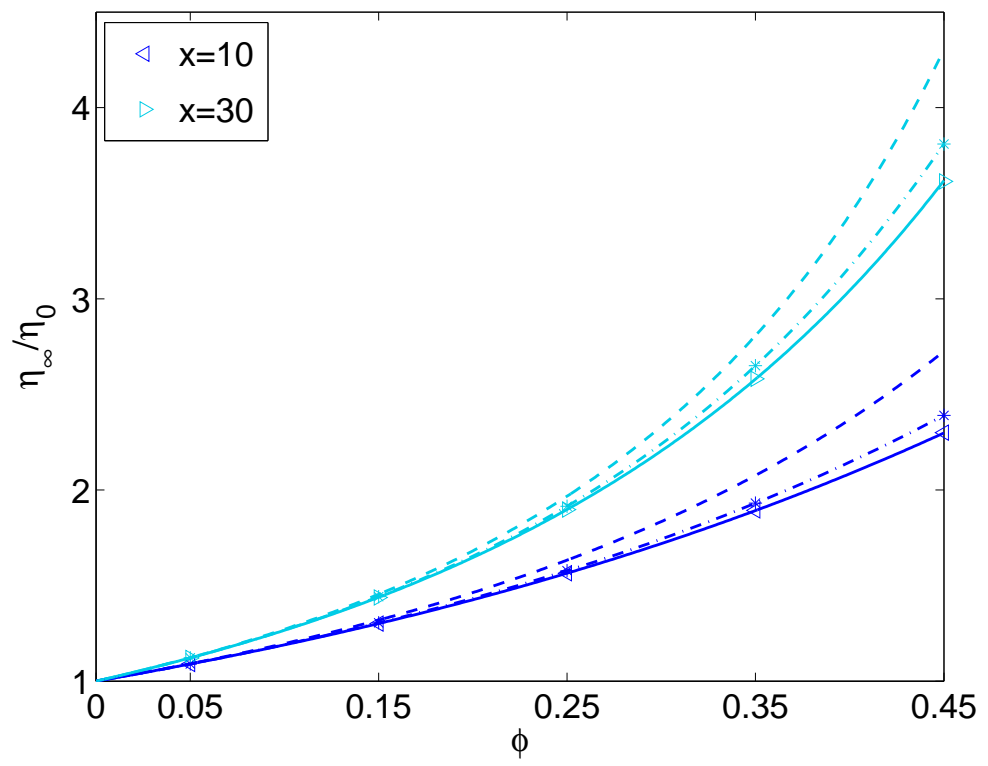

FIG. 4: High-frequency viscosity simulation results (symbols, connected by solid lines which correspond to the Saitô fitting formulas (11) and (14)) versus the ERM and the HRM predictions (dashed and dashed-dotted lines, respectively).

We can use the high-frequency viscosity data for porous particles summarized in table I to quantify the performance of the ERM at higher volume fractions. In Fig. 4, simulation data for $\eta_{\infty}$ are compared to the ERM prediction for $\eta_{\infty}$ at various porosity values. The comparison reveals the poor performance of the ERM for intermediate values $x \sim 10-30$. Clearly, there is a demand for a better effective radius model. In the following, an improved model will be discussed.

\section{B. Hydrodynamic radius model}

In the effective radius model discussed above, two effective (non-porous) particles are allowed to approach each other up to the center-to-center distance $2 a_{\text {eff }}<2 a$. However, 
the rigid skeleton of the actual porous particles of radius $a$ does not allow them to overlap, and the statistical distribution function of the effective particles should take this into account. We present now an improved model where the non-overlap is obeyed, referred to as the hydrodynamic radius model (HRM). In this model, the porous spheres of radius $a$ are described in a better approximation as spherical annulus particles consisting of a dry core of effective hydrodynamic radius $a_{\mathrm{eff}}(x)$, and an outer excluded volume radius $a$, which enters into the statistical distribution through the no-overlap requirement (see Fig. 3). In this refined model, the direct interactions of the actual porous particles are not changed, so that $g_{0}(r)=\theta(r-2 a)$.

In the HRM, the Huggins coefficient is evaluated from Eqs. (9)-(10) for a system of nonporous hard spheres of radius $a_{\mathrm{eff}}(x)$, but with $g_{0}(r)$ specified as $\theta(r-2 a)$. Consequently, the Huggins coefficient in this model is given by

$$
k_{H}^{\mathrm{HRM}}(x)=\frac{2}{5}+\frac{6}{5 a_{\mathrm{eff}}^{3}} \int_{2 a}^{\infty} J^{\mathrm{hs}}(r) r^{2} d r=k_{H}^{\mathrm{hs}}-\frac{6}{5 a_{\mathrm{eff}}^{3}} \int_{2 a_{\mathrm{eff}}}^{2 a} J^{\mathrm{hs}}(r) r^{2} d r,
$$

with $k_{H}^{\mathrm{hs}}=k_{H}(\infty)$. Here, $J^{\mathrm{hs}}(r)=J\left(r / a_{\mathrm{eff}}, \infty\right)$ is Batchelor and Green's function for nonporous hard spheres (hs) of radius $a_{\text {eff }}$ defined in Eq. (17). Through this radius, $k_{H}^{\text {hs }}$ becomes implicitly dependent on the reduced penetration depth $x$. Unlike the ERM, the Huggins coefficient in the HRM has the desired feature of being smaller, for finite $x$, than the zeroporosity Huggins coefficient $k_{H}^{\text {hs }}$. The calculated values of $k_{H}^{\mathrm{HRM}}(x)$ are listed in table IV, together with the corresponding effective hydrodynamic radius.

TABLE IV: HRM-predicted Huggins coefficient, $k_{H}^{\mathrm{HRM}}(x)$, and effective hydrodynamic radius $a_{\text {eff }}(x)$, as functions of $x$ as indicated.

\begin{tabular}{|c|c|c|c|c|c|c|}
\hline$\backslash x$ & 5 & 10 & 20 & 30 & 50 & 100 \\
\hline$a_{\mathrm{eff}} / a$ & 0.755 & 0.880 & 0.943 & 0.963 & 0.979 & 0.990 \\
\hline$k_{H}^{\mathrm{HRM}}$ & 0.562 & 0.658 & 0.723 & 0.747 & 0.768 & 0.784 \\
\hline
\end{tabular}

The comparison with the high-precision values for $k_{H}(x)$ in table I reveals that, for $x \geq 5$, 
the accuracy of the HRM Huggins coefficient is better than $6 \%$, and for $x>30$ even better than $2 \%$.

For higher volume fractions, the HYDROMULTIPOLE numerical code is applied to compute $\eta_{\infty}$ in the HRM-model. The resulting values for $\eta_{\infty}$ are listed in Table V. They are significantly better than those obtained by the ERM, as illustrated in Fig. 4.

TABLE V: High-frequency viscosity prediction, $\eta_{\infty}^{\mathrm{HRM}} / \eta_{0}$, obtained from the HYDROMULTIPOLE simulations performed for the HRM model.

$$
\begin{array}{lcccccc}
\phi \backslash x & 5 & 10 & 20 & 30 & 50 & 100 \\
& & & & & & \\
\hline & & & & & & \\
0.05 & 1.055 & 1.090 & 1.113 & 1.122 & 1.129 & 1.134 \\
0.15 & 1.177 & 1.305 & 1.403 & 1.442 & 1.475 & 1.500 \\
0.25 & 1.315 & 1.578 & 1.812 & 1.915 & 2.01 & 2.08 \\
0.35 & 1.471 & 1.931 & 2.41 & 2.65 & 2.87 & 3.08 \\
0.45 & 1.648 & 2.39 & 3.30 & 3.81 & 4.36 & 4.90
\end{array}
$$

Calculated values of the high-frequency viscosity, $\eta_{\infty}^{\mathrm{HRM}}$, predicted by the HRM model and calculated using the HYDROMULTIPOLE program are listed in table $\mathrm{V}$ for a wide range of $\phi$ and $x$ values. The price to pay for the improved accuracy of the HRM is that $\eta_{\infty}^{\mathrm{HRM}}$ must be computed numerically. However, to this end one can use a standard hydrodynamic multipole force method for non-porous spheres. If one is satisfied with somewhat less accuracy, one can use instead the generalized Saiô formula with Eq. (12) for $S_{1}(x, \phi)$ and $[\eta]=5 / 2$, and $k_{H}^{\mathrm{HRM}}(x)$ taken from table IV.

\section{CONCLUSIONS}

We have analyzed the porosity and concentration dependence of the high-frequency shear viscosity in a suspension of uniformly porous spheres with excluded volume interactions. The flow inside the particles was described by the Debye-Bueche-Brinkman equation with 
the uniform permeability. Porous particles can be often characterized approximately by a uniform mean porosity. Precise values for $\eta_{\infty}$ have been calculated using a simulation method encoded in our HYDROMULTIPOLE program extended to porous particles. The influence of porosity on $\eta_{\infty}$ is found to be significant, in particular at larger volume fractions, and is thus amenable to experimental detection.

A virial expansion of $\eta_{\infty}$ was performed on the basis of our simulation data up to second order in the volume fraction. The virial expansion result was used to derive two generalized and easy-to-use generalized Saitô formulas given by Eq. (11) in conjunction with Eqs. (12) and (14) for the Saitô functions $S_{1}(x, \phi)$ and $S_{3}(x, \phi)$, respectively. The two generalized Saitô formulas are in distinctly better agreement with the simulation data for $\eta_{\infty}$ than the corresponding truncated virial series. The Saitô formula for $\eta_{\infty}$ based on $S_{1}(x, \phi)$, which uses the Huggins coefficient as the only non-trivial input, is already in decently good overall agreement with the simulation data, but there are noticeable differences at intermediate values of $x$. The generalized Saitô formula with the third-order polynomial in $\phi, S_{3}(x, \phi)$, fitted to the simulations, accurate for $\phi \leq 0.45$, can be also extended to the complete fluid-phase regime. Table III gives the coefficients of the polynomial for an extended, representative set of porosity values.

We have studied the performance of two effective models of porous spheres. The effective radius model is most simple in its application, since $\eta_{\infty}$ follows straightforwardly using a known analytic expression for $\eta_{\infty}\left(\infty, \phi_{\text {eff }}(x)\right)$. The accuracy of the ERM, however, is poor for a typical permeability with $x=10-30$. A more precise hydrodynamic radius model has been proposed. In this second model, a porous sphere is replaced by an effective particle with a smaller, non-porous core representing its hydrodynamic influence. The direct interactions of the original porous sphere are kept unchanged. The HRM is straightforwardly applicable to the calculation of short-time diffusion properties properties and the sedimentation coefficient of excluded-volume porous spheres, and to porous particles with (additional) soft direct interactions. Porosity is less influential for particles with longer-ranged soft repulsion since near-contact configurations are unlikely. Different from this, the effect of porosity on $\eta_{\infty}$ is enhanced in the presence of attractive interactions.

Finally, we point out that knowledge on short-time dynamic properties is indispensable for a better understanding of corresponding long-time dynamic properties, such as the static viscosity. Short-time transport coefficients have been used, e.g., as input in mode-coupling 
and dynamic density functional theory calculations of long-time properties. For neutral colloidal spheres with hard-core direct interaction, it has been found empirically that a simple rescaling of long-time diffusion and viscosity quantities, calculated more simply without HIs, by their short-time analogues with HIs, allows for decent estimates of the long-time quantities in the presence of HIs. These short-time hydrodynamic rescaling prescriptions work better at larger concentrations where significant dynamic caging of particles by neighboring ones occurs [53, 61-63]. For neutral hard spheres and charged spheres alike, an approximate time-factorization relation for the dynamics structure factor measured in dynamic scattering experiments has been found, relating wavenumber-dependent short-time and long-time diffusion functions [64]. In place of using approximate scaling relations, it would be most satisfying to obtain long-time properties in concentrated systems, with many-body HIs included, from first-principles calculations based on the generalized Smoluchowski equation or the equivalent overdamped Langevin equation. Such calculations, e.g., of the static viscosity of porous spheres, are very difficult to date but have become feasible with our simulation method and can be the subject of a future work.

\section{Acknowledgments}

G.N., M.L.E.J. and B.C. acknowledge support by the Deutsche Forschungsgemeinschaft (SFB-TR6, project B2). M.L.E.J., E.W. and G.C.A. were supported in part by the Polish Ministry of Science and Higher Education grant 45/N-COST/2007/0; M.L.E.J. and E.W. also by the COST P21 Action "Physics of droplets". The work of G.C.A. was supported by CAPES Foundation/Ministry of Education of Brazil. Numerical calculations were done at the Academic Computer Center in Gdańsk, Poland.

[1] A. A. Zaman and C. S. Dutcher, J. Am. Ceram. Soc. 89, 422 (2006).

[2] R. Tao, Int. J. Mod. Phys. B 21, 4767 (2007).

[3] T. Eckert and W. Richtering, J. Chem. Phys. 129, 124902 (2008).

[4] R. Sigel et al., Phys. Rev. Lett. 83, 4666 (1999).

[5] M.H.G. Duits, P. A. Nommensen, D. van den Ende, and J. Mellema, Colloids Surf. A 183-185, 
335 (2001).

[6] G. Mo and A. S. Sangani, Phys. Fluids 6, 1637 (1994).

[7] A.A. Potanin and W.B. Russel, Phys. Rev. E 52, 730 (1995).

[8] S.L. Elliot and W.B. Russel, J. Rheol. 42, 361 (1998).

[9] P. A. Nommensen, M. H. G. Duits, D. van den Ende, and J. Mellema, Phys. Rev. E 59, 3147 (1999).

[10] A.J.C. Ladd, J. Chem. Phys. 93, 3484 (1990).

[11] P. N. Segrè, O. P. Behrend and P. N. Pusey, Phys. Rev. E 52, 5070 (1995).

[12] J.F. Brady, Curr. Opin. Colloid Interface Sci. 1, 472 (1996).

[13] A. Sierou and J.F. Brady, J. Fluid Mech. 448, 115 (2001).

[14] A.J. Banchio and J.F. Brady, J. Chem. Phys. 118, 10323 (2003).

[15] A.J. Banchio and G. Nägele, J. Chem. Phys. 128, 104903 (2008).

[16] C.I. Mendoza and I. Santamaria-Holek, J. Chem. Phys. 130, 044904 (2009).

[17] Z. Chen, J. Zhu, P. Chaikin, S.-E. Phan and W.R. Russel, Phys. Rev. E 65041405 (2002).

[18] S.B. Chen, Phys. Fluids 10, 1550 (1998).

[19] P.G. Wolynes and J.A. McCammon, Macromolecules 10, 86 (1977).

[20] N.J. Wagner and J.F. Brady, Physics Today, October 2009 issue, 27-32 (2009).

[21] H. Ohshima, Colloids and Surfaces A: PhysicoChem. Eng. Aspects 347, 33 (2009).

[22] H. Ohshima, Theory of Colloid and Interface Electric Phenomena, Elsevier, Amsterdam (2006).

[23] V. Natraj and S.B. Chen, J. Colloid Interface Sci. 251, 200 (2002).

[24] E. Ruiz-Reina and F. Carrique, J. Phys. Chem. C 111, 141 (2007).

[25] G.C. Abade, B. Cichocki, M.L. Ekiel-Jeżewska, G. Nägele and E. Wajnryb, submitted.

[26] J. Bergenholtz, F.M. Horn, W. Richtering, N. Willenbacher and N.J. Wagner, Phys. Rev. E 58, R4088 (1998).

[27] J. Bergenholtz, N. Willenbacher, N.J. Wagner, B. Morrison, D. van den Ende and J. Mellema, J. Colloid Interface Sci. 202, 430 (1998).

[28] T. Shikita and D.S. Pearson, J. Rheol. 38, 601 (1994).

[29] G. Fritz, B.J. Maranzano, N.J. Wagner and N. Willenbacher, J. Non-Newtonian Fluid Mech. 102, 149 (2002).

[30] F.M. Horn, W. Richtering, J. Bergenholtz, N. Willenbacher and N.J. Wagner, J. Colloid 
Interface Sci. 225, 166 (2000).

[31] G.H. Koenderink, H. Zhang, D.G.A.L. Aarts, M.P. Lettinga, A.P. Philipse and G. Nägele, Faraday Discuss. 123, 335 (2003).

[32] H.C. Brinkman, Appl. Scient. Res. A1 27, 81 (1947).

[33] H.C. Brinkman, Proc. Acad. Sci. Amsterdam 50, 618 (1947).

[34] H.C. Brinkman, Physica 13, 447 (1947).

[35] H.C. Brinkman, Proc. R. Dutch Acad. Sci. 50, 618, 821 (1947).

[36] P. Debye, Phys. Rev. 71, 486 (1947).

[37] P. Debye and A.M. Bueche, J. Chem. Phys. 16, 573 (1948).

[38] J.J.L. Higdon and M. Kojima, Int. J. Multiphase Flow 7, 719 (1981).

[39] B. Cichocki, B. U. Felderhof and R. Schmitz, PhysicoChemical Hydrodynamics 10, 383 (1988).

[40] B. Cichocki, B.U. Felderhof, K. Hinsen, E. Wajnryb, and J. Bławzdziewicz, J. Chem. Phys. 100, 3780 (1994).

[41] B. Cichocki, R.B. Jones, R. Kutteh and E. Wajnryb, J. Chem. Phys. 112, 2548 (2000).

[42] B. Cichocki, M.L. Ekiel-Jeżewska and E. Wajnryb, J. Chem. Phys. 111, 3265 (1999).

[43] G.C. Abade, B. Cichocki, M.L. Ekiel-Jeżewska, G. Nägele and E. Wajnryb, J. Chem. Phys. 132, 014503 (2010).

[44] G.C. Abade, B. Cichocki, M.L. Ekiel-Jeżewska, G. Nägele and E. Wajnryb, Phys. Rev. E 81, 020401(R) (2010).

[45] S. Kim and S. J. Karrila, Microhydrodynamics, Dover Publications, Mineola, 2005.

[46] P. Szymczak and B. Cichocki, J. Stat. Mech.: Theory Exp., P0125 (2008).

[47] B. Cichocki, M.L. Ekiel-Jeżewska and E. Wajnryb, J. Chem. Phys. 119, 606 (2003).

[48] B. Cichocki, B.U. Felderhof and R. Schmitz, Physica A 154, 233 (1989).

[49] G.K. Batchelor and J.T. Green, J. Fluid Mech. 56, 401 (1972).

[50] W.B. Russel, J. Chem. Soc. Faraday Trans. 2 80, 31 (1984).

[51] B. Cichocki and B.U. Felderhof, J. Chem. Phys. 89, 1049 (1988).

[52] B. Cichocki and B. U. Felderhof, J. Chem. Phys. 130, 164712 (2009).

[53] A.J. Banchio, J. Bergenholtz and G. Nägele, Phys. Rev. Lett. 82, 1792 (1999).

[54] W. van Saarloos, Physica 147A, 280 (1987).

[55] L. Gmachowski, Colloids and Surfaces A: Physicochem. Eng. Aspects, 215, 173 (2003).

[56] B.U. Felderhof and J.M. Deutch, J. Chem. Phys. 62, 2391 (1975). 
[57] B.U. Felderhof and R.B. Jones, Physica A 93, 457 (1978).

[58] R.E. Larson and J.J.L. Higdon, J. Fluid Mech. 166, 449 (1987).

[59] A.S. Sangani and S. Behl, Phys. Fluids A 1, 21 (1988).

[60] N. Lecoq, R. Anthore, B. Cichocki, P. Szymczak and F. Feuillebois, J. Fluid Mech. 513, 247 (2004).

[61] M. Medina-Noyola, Phys. Rev. Lett. 60, 2705 (1988).

[62] J.F. Brady, J. Chem. Phys. 99, 567 (1993).

[63] E. Wajnryb, P. Szymczak and B. Cichocki, Physica A 335, 339 (2004).

[64] P. Holmqvist and G. Nägele, Phys. Rev. Lett. 104, 058301 (2010). 